\title{
Policy and Regulation in Digital Health
}

\author{
Fazilah Shaik Allaudin
}

Ministry of Health Malaysia, Federal Government Administrative Centre, Federal Territory of Putrajaya

\begin{abstract}
The dawn of the Fourth Industrial Revolution and the advances in telecommunication are presenting multiple strategies and challenges ahead which includes policy and regulation, clinical evidence, safety and quality, medical ethics and clinical practice. However, these disruptions are also creating new opportunities to solve some of the biggest health-related challenges facing the world today. The democratization of care and the great potential to health care offered by the digital revolution is the new paradigm embracing the delivery of care. The deployment of these new technologies and advances is progressing faster than regulatory framework can react. At the same time new technologies can deepen healthcare inequalities and unaffordability. Since 2018, efforts are underway by MOH to review and develop regulatory strategies and policies to foster digital health technologies, advocate translational research, spur innovations and boost the digital economy while at the same time, building an enabling ecosystem in Malaysia. New service models, technologies and innovations must be based on the principles of value-based, safety, quality, confidentiality, accountability and traceability. The safe practice of digital health should keep the consumers of health care at the heart of the adoption.
\end{abstract}

